\title{
L'erreur serait de refuser de parler ouvertement, sereinement, du rationnement - Que nous apprend l'expérience néo-zélandaise?
}

C'est volontiers que nous faisons quelques commentaires sur le texte de Mme Wendy Edgar (voir pages 190 à 194). Rappelons à ce propos qu'elle a été invitée en août 1999 à participer à plusieurs réunions en Suisse sur le thème du rationnement, notamment sous les auspices de la Fédération des médecins suisses (FMH) et de la Société suisse pour la politique de la santé.

D’abord, remarque générale, il faut dire que les termes utilisés peuvent avoir une influence indue. Ainsi, en Suisse aussi, tout le monde (les politiques, les professionnels, les média) est d'accord pour dire qu'il est nécessaire de fixer des priorités en ce qui concerne l'offre de soins de santé, et ceci d'autant plus en période de fortes contraintes sur les budgets publics. Or, en réalité, établir des priorités est synonyme de limiter la dissémination des services, ce qui correspond à rationner, un mot qui fait peur (pour des raisons historiques que par ailleurs on peut comprendre). Ainsi, pour parler d'une problématique semblable, un terme est bien accepté alors que l'autre serait considéré comme tabou; il convient de sortir de ces pièges terminologiques liés à la connotation positive ou négative de certaines expressions.

S'agissant de la Nouvelle Zélande, il est important de voir comment on y a entrepris, dès le début des années 1990, un travail de réflexion puis la mise en ouvre de mesures dans un sens explicite de rationnement. Ceci en reconnaissant l'écart inévitable entre l'éventail toujours croissant des prestations médicales et techniques qui peuvent être offertes à des personnes malades, ou d̀ des groupes à risque, et la relative stagnation des ressources consacrées $\dot{a}$ financer ces prestations. Il est clair que cette situation est en train de se concrétiser aussi dans un pays aussi prospère que la Suisse, dont les citoyens ont été habitués jusqu'ici à des prestations particulièrement étoffées, fournies sans délais d'attente.
Ce qui frappe, c'est qu'un pays de culture anglosaxonne lavec ce que cela représente de respect des droits de la personne - historiquement bien avant la Suisse, la France ou l'Allemagne), de tradition chrétienne, pose ouvertement devant la population le défi d'adapter les objectifs du système de santé aux moyens disponibles. Et, même si cela pose des questions éthiques et pratiques lourdes, il apparaît que les Néo-Zélandais admettent, de bon ou de moins bon gré, qu'il en soit ainsi. C'est un enseignement d'importance, qui relativise certains discours sur le caractère qui serait forcément inacceptable de discuter ce sujet dans une société civilisée. En fait, dire que le débat sur le rationnement est inacceptable, c'est dire qu'on refuse la réalité! Pourtant, la réalité est là: il ne peut faire de doute que le fossé déjà mentionné entre la multiplication des offres possibles et les limites de la capacité à financer obligera à cette réflexion. Si elle n'est pas faite de manière ouverte, alors c'est le rationnement lui-même qui sera introduit sans dire son nom, principalement sans doute sur la base de la capacité des patients à payer (à payer plus!), qui est la plus mauvaise manière de rationaliser ou rationner: à l'évidence en effet, l'établissement de priorités dans les soins doit éthiquement être basé sur les besoins médicaux des personnes et pas sur leurs ressources financières.

Il est vrai que le politiquement correct qui prévaut en Suisse frissonne à la lecture de phrases de Mme Edgar comme: "Les personnes se trouvant en liste d'attente et qui ne satisfont pas aux critères établis sont informées clairement qu'elles ne recevront pas pour l'instant de soins financés publiquement, et elles sont orientées vers leur médecin généraliste." Ou encore: "Le succès de la technologie médicale a créé un dilemme auquel nous devons tous faire face - le mythe que la mort n'est qu'une option (facultative), que chaque vie peut et doit être sauvée si la technologie existe, quelle que soit la qualité de vie prévisible (suite au traitement) ou le coût du traitement." 
Aujourd'hui, nos moyens financiers permettent en Suisse d'être moins drastique qu'en Nouvelle Zélande. Heureusement. L'essentiel cependant est qu'il ne saurait $y$ avoir de différence fondamentale, de nature, entre ce qui se passera chez nous dans les années à venir et ce qui se passe aux antipodes. Nous croyons impératif de ne pas faire usage d'écrans de fumée ou idéologiques. A cet égard, le propos iconoclaste d'un observateur averti de la scène médicosanitaire suisse, Secrétaire général de la Société suisse pour la politique de la santé, retient l'attention: "Comme tous les pays, nous avons déjà ce qu'on appelle une médecine à plusieurs vitesses. Celui qui ne le remarquerait pas souffre de troubles de perception (...). Un système de rationnement sérieux et démocratique ne conduit pas à une médecine à plusieurs vitesses, mais au contraire l'empêche. Un tel système peut assurer le traitement équitable des faibles: personnes âgées, pauvres, ceux qui sont moins instruits, les personnes seules, les patients avec des maladies particulières (...). Presque tous les critiques de la notion de rationnement s'en rendent compte après une discussion explicative d'une demi-heure: ce n'est pas celui qui refuse de parler de rationnement ou l'écarte comme un tabou qui a une attitude sociale, mais c'est bien celui qui s'engage pour que le rationnement soit établi de manière aussi équitable, démocratique et ouverte que possible. C'est le fait de nier la problématique du rationnement qui est non éthique et antisocial." [1]
Fortes paroles, encore moins politiquement correctes que celles de Mme Edgar dans la mesure où elles émanent d'un compatriote. Cela étant, et audelà de telle ou telle formule-choc, nous pensons aussi que, du point de vue de la prestation de soins de santé adéquats à l'ensemble des 7 millions de résidents de la Suisse, refuser d'entrer en matière sur la question du rationnement aura à terme des conséquences plus délétères que d'en parler dès maintenant, y compris et notamment sur la place publique. Aussi factuellement et aussi sereinement que possible, malgré le fait que nous soyons, en tant que membres des professions médicales et soignantes, particulièrement touchés par les perspectives que ce débat ouvre. Cela demande une bonne dose de ce que nos compatriotes alémaniques appellent "Zivilcourage»; cela demande l'émergence d'un nouvel esprit civique, chez les responsables publics et professionnels comme chez les citoyens-assurés-patients.

Dr Jean Martin, PD Médecin cantonal, Lausanne

1 Kocher G. Editorial. GPI/Gesundheitspolitische Informationen. 1999;2. (notre traduction). 